\title{
Rates of Convergence for Viscous Splitting of the Navier-Stokes Equations
}

\author{
By J. Thomas Beale* and Andrew Majda**
}

\begin{abstract}
Viscous splitting algorithms are the underlying design principle for many numerical algorithms which solve the Navier-Stokes equations at high Reynolds number. In this work, error estimates for splitting algorithms are developed which are uniform in the viscosity $\nu$ as it becomes small for either two- or three-dimensional fluid flow in all of space. In particular, it is proved that standard viscous splitting converges uniformly at the rate $C \nu \Delta t$, Strang-type splitting converges at the rate $C \nu(\Delta t)^{2}$, and also that solutions of the Navier-Stokes and Euler equations differ by $C \nu$ in this case. Here $C$ depends only on the time interval and the smoothness of the initial data. The subtlety in the analysis occurs in proving these estimates for fixed large time intervals for solutions of the Navier-Stokes equations in two space dimensions. The authors derive a new long-time estimate for the two-dimensional NavierStokes equations to achieve this. The results in three space dimensions are valid for appropriate short time intervals; this is consistent with the existing mathematical theory.
\end{abstract}

1. Introduction. Many of the most challenging and interesting numerical calculations in fluid dynamics involve the approximation of solutions of the NavierStokes equations at sufficiently high Reynolds numbers. (We will refer to "small viscosity" and "high Reynolds number" interchangeably via standard nondimensionalization.) The random vortex method introduced by Chorin [2] for high Reynolds number flows in two space dimensions is a particularly successful numerical method for this class of problems with some unusual mathematical features. The method is a fractional step algorithm: in the first step, the inviscid Euler equations are approximated by a vortex-blob method; in the second step, the effects of small viscosity are simulated by vorticity creation at the boundary and a random walk of the blobs. Recently, questions have been raised as to the soundness of the above random vortex method even as a numerical method for computing solutions of the Navier-Stokes equations defined on all of two-dimensional space, so that boundary layers do not occur; e.g., see [9]. The main results of this paper provide a rigorous proof of the fact that the viscous splitting algorithms, which are the underlying design principle for the random vortex method, converge to solutions of the Navier-Stokes equations in all of space at a rate which improves as the viscosity becomes smaller. The precise quantitative rate is given in the theorem stated below after these algorithms have been defined.

Solutions of the Navier-Stokes equations are given by a velocity field $u^{\nu}(x, t)$, $x \in \mathbf{R}^{N}, N=2,3,0 \leqslant t \leqslant T$, which satisfies the differential equations and initial

1980 Mathematics Subject Classification. Primary 65M15, 76D05.

Key words and phrases. Splitting algorithms, Navier-Stokes equations, Euler's equations.

* Partially supported by N.S.F. Grant MCS-78-00908.

** Partially supported by N.S.F. Grant MCS-79-02735. 
conditions

$$
\begin{aligned}
& \frac{\partial u^{\nu}}{\partial t}+u^{\nu} \cdot \nabla u^{\nu}=\nu \Delta u^{\nu}-\nabla p^{\nu} \\
& \nabla \cdot u^{\nu}=0, \quad u^{\nu}(x, 0)=u_{0}(x)
\end{aligned}
$$

where $\nu$ is the viscosity, normalized so that $0 \leqslant \nu \leqslant 1$, and $p^{\nu}$ is the corresponding scalar pressure. To discuss the viscous splitting algorithms which we analyze below, we will write $E(t)$ for the solution operator of Euler's equations; i.e., if $v_{0}$ is a divergence-free vector field on $\mathbf{R}^{N}, E(t) v_{0}=v(t)$, where $v(t)$ satisfies Euler's equations

$$
\begin{gathered}
v_{t}+v \cdot \nabla v+\nabla p=0 \\
\nabla \cdot v=0, \quad v(0)=v_{0}
\end{gathered}
$$

We denote by $u^{0}(t)$ the solution of (1.2) with initial data $u_{0}(x)$. Similarly, $H(t)$ will be the solution operator for the heat equation with small viscosity $\nu$; thus, $H(t) w_{0}=w(t)$ where

$$
w_{t}=\nu \Delta w, \quad w(0)=w_{0} .
$$

If $w_{0}$ is a divergence-free vector field, then $H(t) w_{0}$ has the same property.

The viscous splitting algorithm, which is the underlying basis for the random vortex method, is given by the approximation

$$
\tilde{u}_{n}=(H(\Delta t) E(\Delta t))^{n} u_{0}
$$

and one expects that $\tilde{u}_{n}$ approximates $u^{\nu}$ at the time $n \Delta t$. We also analyze another similar viscous splitting algorithm below which has improved rates of convergence and is defined by

$$
u_{n}=(H(\Delta t / 2) E(\Delta t) H(\Delta t / 2))^{n} u_{0}
$$

(This algorithm is motivated by Strang's remarks in [10].)

We introduce $|\cdot|_{s}$ (or sometimes $|\cdot|_{H^{s}}$ for emphasis) for the $L^{2}$-Sobolev norm of order $s$ of a given function and say $u \in H^{s}$ when $|u|_{s}<\infty$. Below, we use the fact that $H^{s}\left(\mathbf{R}^{N}\right)$ is a Banach algebra under pointwise multiplication for $s \geqslant 2$ provided that $N=2,3$; see [6]. Our main result is the following one.

THEOREM (Improved rates of convergence as the viscosity tends to zero).

(A) For $N=2$, suppose $T$ is an arbitrarily large time and $s$ is a fixed nonnegative integer.

Assume $u_{0} \in H^{s+\sigma}$, where $\sigma$ is a fixed integer determined below, and also assume $\nabla \times u_{0} \in L^{1}\left(\mathbf{R}^{2}\right)$. Then

$$
\begin{gathered}
\max _{0<n \Delta t<T}\left|u^{\nu}(n \Delta t)-\tilde{u}_{n}\right|_{s} \leqslant C_{1} \nu \Delta t, \\
\max _{0<n \Delta t<T}\left|u^{\nu}(n \Delta t)-u_{n}\right|_{s} \leqslant C_{2} \nu(\Delta t)^{2}, \\
\max _{0<t<T}\left|u^{\nu}(t)-u^{0}(t)\right|_{s-2} \leqslant C_{3} \nu, \quad s \geqslant 4,
\end{gathered}
$$

where the constants $C_{i}, i=1,2$, are independent of $\nu$ and depend only on $\left|u_{0}\right|_{s+\sigma}, T$, and $\left|\nabla \times u_{0}\right|_{L^{1}}$. The constant $C_{3}$ depends only on $T,\left|u_{0}\right|_{s}$, and $\left|\nabla \times u_{0}\right|_{L^{1}}$. 
(B) For $N=3$, the same results as in (A) are valid provided that the time interval $T$ is restricted to a sufficiently small size determined in [6].

We note that all the statements above hold with obvious modifications for spatially periodic flows of fixed periods in each coordinate direction. A precise version of the above theorem is given by Theorems 1.1-1.3, stated below. Some background material regarding the mathematical properties of solutions of the Navier-Stokes and Euler equations, which is useful for understanding the above theorem, and which we use freely below, is contained in the references [6] and [8]. In [8], McGrath constructed classical solutions of the Navier-Stokes and Euler equations in $\mathbf{R}^{2}$ for all time and proved the convergence, without a rate, of $u^{\nu}(t)$ to $u^{0}(t)$ as $\nu$ tends to zero; in [6], Kato proved a similar result in $\mathbf{R}^{3}$ for sufficiently short time intervals, and the same facts are also valid, mutatis mutandis, for flows in $\mathbf{R}^{2}$. Earlier, Ebin and Marsden [3], [4] studied the Euler equations in Lagrangian coordinates with $\mathbf{R}^{N}$ replaced by a compact manifold. They proved the convergence of the algorithm defined by $\tilde{u}_{n}$ to $u^{\nu}$, without a rate, for sufficiently short time intervals when $N=2$ or 3 . The subtlety in the proof of the above theorem lies in the proof of (A) for arbitrarily long time intervals in two space dimensions; in fact, in the course of proving (A) for large $s$, we derive a new estimate for solutions of (1.1) when $N=2$ (see (1.8) below).

Part (3) of the above theorem is a rigorous mathematical statement of the fact that the Navier-Stokes equations are a regular perturbation of the Euler equations when no boundaries are present. This fact is discussed in Section 4, but, for the moment, we remark on some of the numerical implications. In [5], Hald established the important result that appropriate inviscid vortex methods in all of $\mathbf{R}^{2}$ converge in $L^{2}$ with second order accuracy to the solution $u^{0}(t)$ of the Euler equation. Denoting these vortex method approximations by $u^{h}(t)$, we have

$$
\max _{0<t<T}\left|u^{h}(t)-u^{0}(t)\right|_{0}<C h^{2} .
$$

From (3) of (A) and the triangle inequality, it follows that

$$
\max _{0<t<T}\left|u^{h}(t)-u^{\nu}(t)\right|_{0} \leqslant C\left(\nu+h^{2}\right)
$$

Thus, even without simulating the effects of viscosity, vortex methods approximate flows in all of $\mathbf{R}^{2}$ with small errors. Chorin [2] conjectured an error of the form $O\left(\nu^{1 / 2}+h^{2}\right)$ for the random vortex method without boundaries; the above estimate indicates that it is extremely unlikely that the addition of a random walk would further deteriorate the accuracy beyond this conjectured bound. These comments should be contrasted with those in [9].

We next discuss an explicit example which indicates that a simulation of the effects of viscosity through splitting algorithms can dramatically improve the quality of the approximate solution even for small viscosity. For flows in $\mathbf{R}^{2}$, we let $\omega=\left(u_{1}\right)_{y}-\left(u_{2}\right)_{x}$ denote the scalar vorticity. Let us assume that, at time $t=0, \omega$ is radially symmetric. It follows in standard fashion [1] that the solution $u^{\nu}$ with this initial data has vorticity given by

$$
\omega^{\nu}=H(t) \omega_{0}
$$


and that

$$
u^{\nu}=H(t) u_{0}
$$

Whenever the initial vorticity is a spherically symmetric function, the solution of the Euler equations is steady, so that $E(t) u_{0}=u_{0}$. From these facts, it follows easily that, for these symmetric initial data,

$$
u_{n} \equiv \tilde{u}_{n} \equiv u^{\nu}(n \Delta t)
$$

so that the viscous splitting algorithms have no error, independent of $\nu$. However, if viscosity is ignored, $E(t) u_{0}=u_{0}$ and the error has the form described in (A3) of the theorem.

For reasons which we discuss below, it is important that we prove the above theorem through the following separate steps. We state Theorems 1.1-1.3 only for the more complicated splitting algorithm in (1.4) since similar and even simpler arguments apply for the algorithm defined by $\tilde{u}_{n}$. Our first result applies to two-dimensional flows when $s=1$ in the above theorem.

THEOREM 1.1. Suppose $u_{0} \in H^{\sigma}\left(\mathbf{R}^{2}\right)$ with $\sigma$ sufficiently large but fixed and also $\nabla \times u_{0} \in L^{1}\left(\mathbf{R}^{2}\right)$. Choose $M>0$ so that

$$
\left|u_{0}\right|_{H^{*}}+\left|\nabla \times u_{0}\right|_{L^{1}} \leqslant M .
$$

For each $\nu$ with $0<\nu \leqslant 1$, let $u^{\nu}$ be the solution of the Navier-Stokes equations (1.1) with initial data $u_{0}$. With $T>0$ arbitrary, and $N$ a positive integer, set $\Delta t=T / N$ and define $u_{n}, 1 \leqslant n \leqslant N$, by (1.4) so that

$$
u_{n}=H(\Delta t / 2) E(\Delta t) H(\Delta t / 2) u_{n-1} \text {; }
$$

then

$$
\max _{1<n<N}\left|u_{n}-u^{\nu}(n \Delta t)\right|_{H^{1}} \leqslant C_{0} \nu(\Delta t)^{2}
$$

where $C_{0}$ depends only on $M$ and $T$.

As mentioned above, for flows in $\mathbf{R}^{3}$, a smooth solution of the Euler or Navier-Stokes equations is known to exist only for some finite time interval depending on the size of the initial data. Assuming $\left|u_{0}\right|_{\sigma}<M$ with $\sigma$ large, the solution $u^{\nu}$ of (1.1), $\nu \geqslant 0$, satisfies

$$
D_{t}\left|u^{\nu}(t)\right|_{\sigma} \leqslant C_{\sigma}\left|u^{\nu}(t)\right|_{\sigma}^{2}
$$

where $C_{\sigma}$ depends on $\sigma$ but not on $\nu$. The solution exists as long as the solution of this inequality remains finite; i.e., for

$$
T_{0}<\left(C_{\sigma} M\right)^{-1}
$$

there is a solution in $C\left(\left[0, T_{0}\right] ; H^{\sigma}\left(\mathbf{R}^{3}\right)\right)$ with

$$
\left|u^{\nu}(t)\right|_{\sigma} \leqslant M\left(1-C_{\sigma} M t\right)^{-1} \equiv \phi_{M}(t) .
$$

This result is due to Kato and may be found in [6].

With this restriction on the time interval, we obtain a result similar to Theorem 1.1. Here, in contrast to Theorem 1.1, we can show the convergence in $H^{m}\left(\mathbf{R}^{3}\right)$ for any $m$ provided $\sigma$ is large enough. The reason is that, for $t \leqslant T_{0}$, (1.7) provides a bound for $u^{\nu}(t)$ independent of $\nu$. 
THEOREM 1.2. Let $m$ be a positive integer. Suppose $u_{0} \in H^{\sigma}\left(\mathbf{R}^{3}\right)$ with $\sigma$ sufficiently large, depending on $m$, and choose $M>0$ so that $\left|u_{0}\right|_{\sigma}<M$. For each $\nu, 0<\nu<1$, let $u^{\nu}$ be the solution of the Navier-Stokes equations with initial data $u_{0}$, defined for $0 \leqslant t \leqslant T_{0}$, where $T_{0}$ satisfies (1.6). With $N$ a positive integer, let $\Delta t=T_{0} / N$, and assume $N$ is large enough so that $2 \Delta t<\left(C_{\sigma} M\right)^{-1}-T_{0}$. If $u_{n}$ is defined by (1.4), $1<n \leqslant N$, then

$$
\max _{1<n<N}\left|u_{n}-u^{\nu}(n \Delta t)\right|_{H^{m}}<C_{0} \nu(\Delta t)^{2}
$$

where $C_{0}$ depends only on $m, M$, and $T_{0}$.

Theorems 1.1 and 1.2 are proved in Section 2, assuming certain lemmas. These lemmas are proved in Section 3. In particular, Lemma 2.1 gives the nonlinear stability of the algorithm in (1.4) in $H^{s}, s>4$, for arbitrarily large time intervals for two-dimensional flows. In Section 4, we use this stability estimate together with Theorem 1.1 to prove the following new estimate for solutions of (1.1) and (1.2):

If $T$ is an arbitrarily large time interval and $u_{0} \in H^{s}\left(\mathbf{R}^{2}\right), \nabla \times u_{0} \in L^{1}\left(\mathbf{R}^{2}\right), s>4$, but otherwise arbitrary, then

$$
\max _{\substack{0<t<T \\ 0<\nu<1}}\left|u^{\nu}(t)\right|_{s} \leqslant C
$$

where $C$ depends only on $T,\left|u_{0}\right|_{s}$, and $\left|\nabla \times u_{0}\right|_{L^{1}}$.

Once the estimate (1.8) is established, it serves as a replacement for (1.7), so that the following facts are proved in Section 4 by essentially a repetition of the argument used in Theorem 1.2.

THEOREM 1.3. Let $\sigma \geqslant 2$ be the integer from Theorem 1.1 and assume that $u_{0} \in H^{s+\sigma}\left(\mathbf{R}^{2}\right), \nabla \times u_{0} \in L^{1}\left(\mathbf{R}^{2}\right), s \geqslant 4$. Then, with the notation of Theorem 1.1,

$$
\begin{gathered}
\max _{0<t<T}\left|u^{\nu}(t)-u^{0}(t)\right|_{s-2} \leqslant C_{1} \nu, \\
\max _{1<n<N}\left|u^{\nu}(n \Delta t)-u_{n}\right|_{s}<C_{2} \nu(\Delta t)^{2},
\end{gathered}
$$

where $C_{2}$ depends only on $T,\left|u_{0}\right|_{s+\sigma}$, and $\left|\nabla \times u_{0}\right|_{L^{1}} ; C_{1}$ has the dependence described in (A3) of the main theorem above.

We remark that the proofs of Theorems 1.1-1.3 do not proceed via the method used by Strang [11] for smooth solutions of nonlinear hyperbolic equations-in particular, we must estimate simultaneously in two different small free parameters, $\nu$ and $\Delta t$. Unlike the argument in [11], it is essential that we substitute the approximation from (1.4) into the Navier-Stokes equations (1.1) and derive an equation for the error in satisfying (1.1). Furthermore, it is essential that the nonlinear stability of the algorithm in (1.4) is valid (see Lemma 2.1); stability of only the linearization, as in Strang's work, would not be sufficient. Finally, we end this section by commenting that the authors do not know a more direct proof of the estimate (1.8) for two-dimensional solutions of the Navier-Stokes equations at large times, i.e., one which does not use the stability of the viscous splitting approximation (1.4) in an essential fashion.

2. Proofs of Theorems 1.1 and 1.2. We will use the orthogonal projection $P$ of $H^{0}\left(\mathbf{R}^{n} ; \mathbf{R}^{n}\right)$ onto divergence-free vectors. For $v \in H^{0},\{(I-P) v\}^{\wedge}(\xi)$ is just the 
projection of $\hat{v}(\xi)$ on $\xi$. It is evident from the transform that $P$ is also an orthogonal projection on $H^{s}$ with the standard inner product. Applying $P$ to the first of Euler's equations (1.2), we have $P(\nabla p)=0$, and thus $v_{t}=B v$, with

$$
B v=-P(v \cdot \nabla v) \text {. }
$$

We note that $P$ commutes with $\Delta$ and $H(t)$.

The proof of Theorem 1.1 will require the following two lemmas, whose proofs we defer to Section 3. We use the notation

$$
X^{s}\left(\mathbf{R}^{2}\right)=\left\{v \in H^{s}\left(\mathbf{R}^{2}\right): \nabla \times v \in L^{1}\left(\mathbf{R}^{2}\right), \nabla \cdot v=0\right\}
$$

with the obvious norm.

Lemma 2.1. Witn $u_{n}$ as in Theorem 1.1 and $s \geqslant 4$, there exists a constant $C_{1}$, depending only on $s, T$, and $\left|u_{0}\right|_{X^{s}}$, so that $\left|u_{n}\right|_{X^{s}} \leqslant C_{1}$.

LEMMA 2.2. For $s \geqslant 5$, the function $F\left(t, v_{0}\right)=E(t) v_{0}$ is $C^{1}$ from

$$
K^{s}(R)=[0, T] \times\left\{v_{0} \in X^{s}:\left|v_{0}\right|<R\right\}
$$

to $H^{s-2}\left(\mathbf{R}^{2}\right)$, and $F, D_{t} F, D_{v_{0}} F$ are bounded by a constant depending only on $s, T, R$. The derivative with respect to $v_{0}$ is the linear operator $d F\left(t, v_{0}\right)$ taking $w_{0}$ to the solution at time $t$ of

$$
w_{t}+P(v \cdot \nabla w)+P(w \cdot \nabla v)=0, \quad w(0)=w_{0}
$$

where $v(t)=E(t) v_{0}$.

Similarly, there exists $s_{0}$ so that for $s \geqslant s_{0}+3, E(t) v_{0}$ is $C^{3}$ from $K^{s}(R)$ to $H^{s-s_{0}}\left(\mathbf{R}^{2}\right)$, and all derivatives are bounded by a constant depending on $s, T, R$.

It is elementary that $H(t) v_{0}$ is $C^{k}$ from $K^{s}(R)$ to $H^{s-2 k}\left(\mathbf{R}^{2}\right)$. Also we have

$$
\left|H(t) v_{0}\right|_{X^{s}} \leqslant\left|v_{0}\right|_{X^{s}}
$$

As a consequence of the two lemmas and this remark, if we choose a fixed $n$ and define

$$
u(t)=H(t / 2) E(t) H(t / 2) u_{n},
$$

then $u$ is $C^{3}$ as a function of $t \in[0, T]$ with values in $H^{\sigma-s_{0}-12}$. Moreover, $D_{t}^{j} u$ is bounded independent of $\Delta t$ and $n, 0 \leqslant j \leqslant 3$.

With $u$ as above, we will first estimate the growth in $H^{0}$ of

$$
r(t)=u(t)-u^{v}\left(t+t_{n}\right)
$$

To do so, we check the consistency of (2.4) with the Navier-Stokes equations. Differentiating (2.4), we have

$$
D_{t} u(t)=(\nu / 2) \Delta H E H u_{n}+H B E H u_{n}+(\nu / 2) H(d E) \Delta H u_{n},
$$

where $H=H(t / 2), E=E(t), d E=d E\left(t ; H(t / 2) u_{n}\right)$. We can rewrite this as

$$
D_{t} u=(\nu \Delta+B) u(t)+f
$$

where $f=f_{1}+f_{2}$ and

$$
f_{1}=[H B-B H] E H u_{n}, f_{2}=(\nu / 2) H[(d E) \Delta-\Delta E] H u_{n} .
$$

Since $u^{v}$ satisfies the same equation with $f$ replaced by 0 , we have

$$
r_{t}=\nu \Delta r+B u-B u^{\nu}+f \text {. }
$$


We can now use (2.1) to express this as

$$
r_{t}=\nu \Delta r-\left(P u^{\nu} \cdot \nabla r\right)-P(r \cdot \nabla u)+f .
$$

Provided $\sigma$ is large enough, $r$ is $C^{1}$ with values in $H^{0}$. Thus, to estimate $r$, we take the inner product of (2.7) with $r$ to obtain

$$
\left(r_{t}, r\right)+\nu|r|_{0}^{2}=-\left(u^{\nu} \cdot \nabla r, r\right)-(r \cdot \nabla u, r)+(f, r) .
$$

The first term on the right is identically zero since $\nabla \cdot u^{\nu}=0$, as can be seen from integration by parts. The second term is bounded by $|u|_{C^{1}}|r|_{H^{0}}^{2}$. Introducing $R(t)=|r(t)|_{H^{0}}$, we have

$$
R_{\imath} \leqslant a R+|f(t)|_{H^{0}}
$$

where $a$ depends only on $M$ and $T$.

We will now estimate $f$. It is obvious that $f_{2}$ is of order $\nu$; to see that the same is true for $f_{1}$, we write $H=H(t / 2)$ as the integral of its derivative. We have $H=I+\nu \bar{H}$, where

$$
\bar{H}=\int_{0}^{t / 2} \Delta H(s) d s .
$$

Then, with $v=E H u_{n}$, we have

$$
\begin{aligned}
f_{1} & =[H B-B H] v=-P H(v \cdot \nabla v)+P[(H v) \cdot \nabla(H v)] \\
& =\nu P\{-\bar{H}(v \cdot \nabla v)+(\bar{H} v) \cdot \nabla(H v)+v \cdot \nabla(\bar{H} v)\} .
\end{aligned}
$$

Thus $f / \nu$ is a $C^{2}$ function of $t$ with values in $H^{1}$, provided $\sigma$ is large enough, and, using Lemma 2.2, we have

$$
\left|f^{\prime \prime}(t)\right|_{H^{1}} \leqslant C \nu, \quad 0 \leqslant t \leqslant \Delta t .
$$

It is obvious that $f(0)=0$; we will show that in fact $f^{\prime}(0)=0$ as well, and it follows that

$$
|f(t)|_{H^{1}} \leqslant C \nu t^{2}, \quad 0 \leqslant t \leqslant \Delta t .
$$

To compute $f^{\prime}(0)$, we first observe that the only contributions come from differentiating the operators inside the commutators; the other terms do not contribute since the commutators are zero at $t=0$. For $f_{1}$, with $v=E H u_{n}$ as before, we have

$$
\begin{aligned}
D_{t}(H B v) & =-P D_{t}[H(v \cdot \nabla v)]=-(\nu / 2) P \Delta(v \cdot \nabla v)+\varepsilon \\
& =-(\nu / 2) P \Delta\left(u_{n} \cdot \nabla u_{n}\right)+\varepsilon,
\end{aligned}
$$

where $\varepsilon$ stands for terms which vanish at $t=0$, or which arise from differentiating $v$ with respect to $t$. In the same way,

$$
\begin{aligned}
D_{t}(-B H v) & =P D_{t}[(H v) \cdot \nabla(H v)] \\
& =(\nu / 2) P\left[u_{n} \cdot \nabla\left(\Delta u_{n}\right)+\left(\Delta u_{n}\right) \cdot \nabla u_{n}\right]+\varepsilon
\end{aligned}
$$

Consequently,

$$
f_{1}^{\prime}(0)=(\nu / 2) P\left\{-\Delta\left(u_{n} \cdot \nabla u_{n}\right)+u_{n} \cdot \nabla\left(\Delta u_{n}\right)+\left(\Delta u_{n}\right) \cdot \nabla u_{n}\right\}
$$

In computing $f_{2}^{\prime}(0)$, we have no contribution from differentiating $d E\left(t ; H(t / 2) u_{n}\right)$ with respect to the second $t$, since $d E(0 ; \cdot) \equiv I$. With $w=H u_{n}$, we have from Lemma 2.2

$$
D_{t}[d E(t ; w) \Delta w]=-P[w \cdot \nabla(\Delta w)+(\Delta w) \cdot \nabla w]+\varepsilon
$$


For the other term in $f_{2}$, we have

$$
-D_{t}[\Delta E w]=-\Delta B w+\varepsilon=\Delta\left(u_{n} \cdot \nabla u_{n}\right)+\varepsilon
$$

Thus

$$
f_{2}^{\prime}(0)=(\nu / 2) P\left\{-u_{n} \cdot \nabla\left(\Delta u_{n}\right)-\left(\Delta u_{n}\right) \cdot \nabla u_{n}+\Delta\left(u_{n} \cdot \nabla u_{n}\right)\right\},
$$

which is precisely $-f_{1}^{\prime}(0)$. We have now shown that $f^{\prime}(0)=0$, and (2.9) is verified.

Combining (2.8) and (2.9), we have the inequality

$$
R^{\prime}(t) \leqslant a R(t)+b v t^{2}
$$

and, for $0 \leqslant t \leqslant \Delta t$,

$$
R^{\prime}(t) \leqslant a R(t)+b \nu(\Delta t)^{2}
$$

so that

$$
R(t) \leqslant e^{a t} R(0)+C \nu(\Delta t)^{2} t, \quad R(\Delta t) \leqslant e^{a \Delta t} R(0)+C \nu(\Delta t)^{3} .
$$

Now let $R_{n}=\left|u_{n}-u^{\nu}(n \Delta t)\right|_{H^{0}}$. We have shown that

$$
R_{k+1} \leqslant e^{a \Delta t} R_{k}+C \nu(\Delta t)^{3} .
$$

Since $R_{0}=0$, it follows inductively that

$$
R_{k} \leqslant C \nu(\Delta t)^{2} a^{-1}\left(e^{a k \Delta t}-1\right)
$$

and therefore

$$
R_{k} \leqslant C^{\prime} \nu(\Delta t)^{2} \text {. }
$$

We have now proved the assertion of the theorem with the error estimated in $H^{0}$, rather than $H^{1}$. To estimate derivatives, we first work with $\nabla \times r$, where the curl is regarded as a scalar, and $r$ is as before. We take the curl of (2.6), noting $\nabla \times(I-P)=0$ and $\nabla \times(u \cdot \nabla u)=(u \cdot \nabla)(\nabla \times u)$. With $\omega=\nabla \times u, \omega^{\nu}=\nabla \times u^{\nu}$, and $\rho=\nabla \times r=\omega-\omega^{\nu}$, we obtain

$$
\begin{aligned}
\rho_{t} & =\nu \Delta \rho-u \cdot \nabla \omega+u^{\nu} \cdot \nabla \omega^{\nu}+\nabla \times f \\
& =\nu \Delta \rho-u^{\nu} \cdot \nabla \rho-r \cdot \nabla \omega+\nabla \times f .
\end{aligned}
$$

Then

$$
\left(\rho_{t}, \rho\right)+\nu|\nabla \rho|_{0}^{2} \leqslant\left|\left(u^{\nu} \cdot \nabla \rho, \rho\right)\right|+|\omega|_{C^{1}}|r|_{H^{0}}|\rho|_{H^{0}}+|f|_{H^{1}}|\rho|_{H^{0}}
$$

The first term on the right is again zero. In the second, $|\omega|_{C^{1}}$ is bounded by a constant if $\sigma$ is large, and, combining (2.10), (2.11), we have

$$
|r|_{H^{0}} \leqslant C \nu(\Delta t)^{2}, \quad 0 \leqslant t \leqslant \Delta t .
$$

Applying (2.9) to the third term, we have

$$
D_{t}|\rho(t)|_{H^{0}}<C \nu(\Delta t)^{2}, \quad 0<t<\Delta t .
$$

Therefore,

$$
|\rho(\Delta t)|_{H^{0}} \leqslant|\rho(0)|_{H^{0}}+C \nu(\Delta t)^{3},
$$

and, by induction, this implies

$$
\left|\nabla \times u_{n}-\nabla \times u^{\nu}(n \Delta t)\right|_{H^{0}} \leqslant C \nu(\Delta t)^{2} .
$$

Now, if $v$ is any divergence-free vector field on $\mathbf{R}^{2}$, we can easily express the first derivatives of $v$ in terms of $\nabla \times v$ in the transform

$$
\left(D_{x_{i}} v\right)^{\wedge}(\xi)=-\xi_{j}|\xi|^{-2}\left(-\xi_{2}, \xi_{1}\right)(\nabla \times v)^{\wedge}(\xi) .
$$


From this it is obvious that

$$
\left|D_{x_{j}} v\right|_{H^{s}} \leqslant|\nabla \times v|_{H^{s}}
$$

for any $s \geqslant 0$. Applying this inequality with $s=0$ to (2.12) and recalling (2.11), we obtain the conclusion of the theorem.

The argument just given applies as well to the three-dimensional case of Theorem 1.2 provided that Lemmas 2.1, 2.2 are replaced with the following modifications.

LEMMA 2.3. Assume $\sigma \geqslant 3$. With $u_{n}$ as in (1.4), we have $\left|u_{n}\right|_{H^{\circ}}<\phi_{M}\left(T_{0}\right)$.

LEMMA 2.4. The statement of Lemma 2.2 holds with $s$ replaced by $\sigma, X^{s}$ by $H^{s}, R$ by $\phi_{M}\left(T_{0}\right)$, and $T$ by $T_{1}=\left\{\left(C_{\sigma} M\right)^{-1}-T_{0}\right\} / 2$.

These lemmas are discussed in Section 3 after Lemmas 2.1 and 2.2. To show convergence in $H^{m}$ in the above argument, we return to Eq. (2.7). Taking the $H^{m}$-inner product with $r$, we have, for $m \geqslant 3$,

$$
\left(r_{t}, r\right)_{m}+\nu(\nabla r, \nabla r)_{m} \leqslant\left|u^{\nu}\right|_{m}|r|_{m}^{2}+C_{1}|u|_{m+1}|r|_{m}^{2}+C_{2}|f|_{m}|r|_{m}
$$

On the right-hand side we have used (2.2) and (2.1) of [6], respectively, in the first two terms. From Lemma 2.3 and the estimate (1.7) for $u^{\nu}$, we have

$$
D_{t}|r(t)|_{m} \leqslant C_{1}|r(t)|_{m}+C_{2}|f(t)|_{m}, \quad 0 \leqslant t \leqslant \Delta t .
$$

Moreover, for $\sigma$ large enough, (2.9) can be improved to

$$
|f(t)|_{m} \leqslant C \nu t^{2}, \quad 0 \leqslant t<\Delta t .
$$

Thus, we obtain for $|r(t)|_{m}$ an estimate of the same form as (2.10) for $R(t)$. The proof of Theorem 1.2 is now completed by repeating the steps which led to (2.11).

For the case of periodic flows, analogous lemmas hold, and the proofs of Theorems 1.1 and 1.2 apply without change. The derivation of the lemmas in this case is discussed at the end of Section 3.

3. Proof of the Lemmas. To prove Lemmas 2.1 and 2.2 we will begin with estimates in classical norms for the solution of Euler's equations as obtained by McGrath [8] and use these to derive estimates in $H^{s}$ for large $s$. In [8] a solution of Euler's equations is constructed on an arbitrary interval $0 \leqslant t \leqslant T_{1}$, using the equation for the vorticity $\omega=\nabla \times u$ of a velocity field $u$ satisfying the two-dimensional Euler equations,

$$
\omega_{t}+u \cdot \nabla \omega=0
$$

The vorticity is treated as the unknown, and the velocity is obtained through an integral operator. The following lemma is a summary of bounds obtained for the solution in [8]. It is assumed that $u(0)$ and $\omega(0)$ are in $C^{1}\left(\mathbf{R}^{2}\right)$ and $\omega(0) \in L^{1}\left(\mathbf{R}^{2}\right)$. Then $u$ and $\omega$ are in $C^{1}\left(\mathbf{R}^{2} \times\left[0, T_{1}\right]\right)$.

LEMMA 3.1. Suppose that

$$
|\omega(0)|_{L^{1}}+|\omega(0)|_{L^{\infty}}=M_{1}
$$

and $\omega(0)$ satisfies the Hölder condition with exponent $\lambda, 0<\lambda<1$, and constant $M_{2} \geqslant 2 M_{1}$. Then, for $0 \leqslant t \leqslant T_{1}$,

(i) $|\omega(t)|_{L^{1}}+|\omega(t)|_{L^{\infty}}=M_{1}$; 
(ii) $\omega(t)$ is Hölder continuous with exponent $\lambda \beta$ and constant $M_{2}$, where $\beta=$ $\exp \left(-C_{0} M_{1} T_{1}\right), C_{0}$ being a universal constant;

(iii) $|u(t)|_{L^{\infty}} \leqslant 2 \pi M_{1}$;

(iv) $\left|D_{x} u(t)\right|_{L^{\infty}} \leqslant C \lambda^{-1}$

(v) $\left|D_{x} \omega(t)\right|_{L^{\infty}} \leqslant\left|D_{x} \omega(0)\right|_{L^{\infty}} \exp \left(C \lambda^{-1} t\right)$.

The constant $C$ depends only on $M_{1}, M_{2}$, and an upper bound for $T_{1}$.

We discuss these statements briefly; lemmas and equations from McGrath's paper referred to here are followed by the label [8]. Assertion (i) follows from (2.3)[8] and the proof of Lemma 2.1[8]. Then (iii) follows from Lemma 1.2(a)[8]. In the definition of $\beta$ on p. 336[8], the constant $L$, according to the proof of Lemma 1.2(c)[8], is a multiple of $M_{1}$. Part (ii) is an obvious improvement of Lemma 2.4[8] in the special case $f=0$. For (iv), we use Lemma 1.2(e)[8] with $\lambda$ replaced by $\lambda \beta$. The proof of that statement is based on the Schauder estimate for the Dirichlet problem. The constant in the Schauder estimate is inversely proportional to the Hölder exponent, so that (iv) results. For (v), we write $\omega$ as in (2.3)[8] with $f=0$ and differentiate to obtain

$$
\left|D_{x} \omega(t)\right| \leqslant\left|D_{x} \omega(0)\right| \cdot\left|D_{x} U_{0 t}\right|,
$$

where $U_{0 t}$ is the flow generated by the velocity, defined in (2.2)[8]. The derivative of $U_{0 t}$ satisfies a variational equation whose coefficients are first derivatives of $u$ (see the bottom of p. 337[8] and Lemma 2.8[8], beginning of proof). Thus

$$
\left|D_{x} U_{0 t}\right| \leqslant \exp \left(C \lambda^{-1} t\right)
$$

using (iv), and this gives (v).

This lemma provides us with bounds on $u$ and $\omega$ in $C^{1}$ for a solution of Euler's equations. The next lemma uses such bounds to estimate $u, \omega$ in Sobolev norms. Norms with subscripts will be Sobolev norms unless indicated otherwise.

LEMMA 3.2. Suppose that for $0 \leqslant t \leqslant T_{1}, u(t)$ and $\omega(t)$ are bounded in $C^{1}\left(\mathbf{R}^{2}\right)$ by a constant $M_{3}$. Suppose $u(0) \in H^{m+1}, m \geqslant 3$, an integer. Then $u \in C\left(\left[0, T_{1}\right] ; H^{m+1}\right)$ $\cap C^{1}\left(\left[0, T_{1}\right] ; H^{m}\right)$. For $0 \leqslant j \leqslant 2$,

$$
|\omega(t)|_{j} \leqslant e^{K_{2} t}|\omega(0)|_{j},
$$

where $K_{2}$ depends only on $M_{3}$. For $3 \leqslant j \leqslant m$,

$$
|\omega(t)|_{j} \leqslant e^{K_{j} t}|\omega(0)|_{j},
$$

where $K_{j}$ depends on $j, M_{3}, T_{1}$, and $|\omega(0)|_{j-1}$. Consequently, for $3 \leqslant j \leqslant m$,

$$
|u(t)|_{j+1} \leqslant C\left\{|u(0)|_{0}+e^{K_{j} t}|\omega(0)|_{j}\right\},
$$

where $C$ depends only on $j$.

Proof. Note first that $|u(t)|_{0}$ is independent of $t$; this is conservation of energy for Euler's equations. Thus, recalling (2.13), under the assumption $u(0) \in H^{0}$, we have $u(t) \in H^{j+1}$ if and only if $\omega(t) \in H^{j}$. The proof will be based on estimates of $\omega(t)$ using the vorticity equation (3.1) and a local existence theorem of Kato [6].

To estimate $\omega(t)$ in $H^{j}$, we will differentiate (3.1). Applying $D_{x}^{\alpha}$, where $\alpha$ is a multi-index, we expect that

$$
D^{\alpha}(u \cdot \nabla \omega)=\sum_{\beta<\alpha} D^{\beta} u \cdot \nabla D^{\alpha-\beta} \omega .
$$


We will first derive estimates on the terms in the expansion which will justify this formula, and which will also be needed for (3.2), (3.3). For the moment we regard $u, \omega$ as arbitrary functions of $x$ with $u \in H^{m+1}, \omega \in H^{m}$. Let $\rho_{\beta}$ be the $\beta$-term on the right in (3.5), and take $|\alpha|=j \leqslant m$. With $\beta=0$, we estimate $\rho_{0}$ in $H^{-1}$, using the fact that multiplication by a $C^{1}$ function is a bounded operator on $H^{-1}$ :

$$
\left|\rho_{0}\right|_{-1} \leqslant C|u|_{C^{\prime}}\left|\nabla D^{\alpha} \omega\right|_{-1}, \quad\left|\rho_{0}\right|_{-1}<C|u|_{C^{\prime}}|\omega|_{j} .
$$

For $|\beta|>0$ we estimate in $H^{0}$. For $|\beta|=1$ or $j$ we have

$$
\begin{array}{cc}
\left|\rho_{\beta}\right| \leqslant C\left|D^{1} u\right|_{C^{0}}|\omega|_{j} & (|\beta|=1), \\
\left|\rho_{\beta}\right| \leqslant C\left|D^{1} u\right|_{j-1}\left|D^{1} \omega\right|_{C^{0}} & (|\beta|=j) .
\end{array}
$$

For $j \geqslant 3$ and $2 \leqslant|\beta| \leqslant j-1$,

$$
\left|\rho_{\beta}\right|_{0} \leqslant C\left|D^{\beta} u\right|_{2}\left|D^{\alpha-\beta+1} \omega\right|_{0}
$$

so that

$$
\left|\rho_{\beta}\right|_{0} \leqslant C\left|D^{1} u\right|_{j}|\omega|_{j-1} \quad(2 \leqslant|\beta| \leqslant j-1) .
$$

We now justify (3.5), assuming $m \geqslant 3$ and $|\alpha| \leqslant m$. We have $|\omega|_{C^{1}} \leqslant C|\omega|_{3}$ and the same for $u$, so that, from (3.6)-(3.9),

$$
\left|\sum_{\beta} \rho_{\beta}\right|_{-1} \leqslant C|\omega|_{m}|u|_{m+1} .
$$

On the other hand, $(u, \omega) \mapsto D^{\alpha}(u \cdot \nabla \omega)$ is continuous, $H^{m+1} \times H^{m} \rightarrow H^{-1}$. Since (3.5) holds for test functions, it also holds for arbitrary $u \in H^{m+1}, \omega \in H^{m}$ by passage to the limit. For later use, we summarize (3.7)-(3.9) using the assumed bound $M_{3}$ and (2.13). As before, we take $j=|\alpha| \leqslant m$. For $j \leqslant 2$, we have from (3.7), (3.8)

$$
\left|\sum_{\beta \neq 0} \rho_{\beta}\right|_{0} \leqslant C_{2} M_{3}|\omega|_{j}
$$

and, for $j \geqslant 3$,

$$
\left|\sum_{\beta \neq 0} \rho_{\beta}\right| \leqslant C_{j}\left\{M_{3}+|\omega|_{j-1}\right\}|\omega|_{j},
$$

$C_{j}$ depending only on $j$.

We now assume that $u$ is in the class stated in the lemma for $0 \leqslant t \leqslant T_{2}$. This is true for some $T_{2}>0$ by a local existence theorem of Kato [6]. Eventually, we will show that we can take $T_{2}=T_{1}$. We will verify (3.2), (3.3) by induction on $j$ for $0 \leqslant t \leqslant T_{2}$. For $j=0$, we multiply the vorticity equation (3.1) by $\omega$ and integrate to find $|\omega(t)|_{0}=|\omega(0)|_{0}$, since $(u \cdot \nabla \omega, \omega)=0$. For $j \geqslant 1$, we apply $D^{\alpha}$ to (3.1), $|\alpha| \leqslant j$, and obtain

$$
\left(D^{\alpha} \omega\right)_{t}+u \cdot \nabla\left(D^{\alpha} \omega\right)=f \equiv-\sum_{\beta \neq 0} \rho_{\beta}
$$

Regarding this as a linear equation in $D^{\alpha} \omega$, we have

$$
D^{\alpha} \omega(x, t)=D^{\alpha} \omega\left(\Phi^{0 t}(x)\right)+\int_{0}^{t} f\left(\Phi^{s t}(x), s\right) d s,
$$

where $\Phi$ is the flow generated by $u$,

$$
D_{s} \Phi^{s t}(x)=u\left(\Phi^{s t}(x), s\right), \quad \Phi^{t t}(x)=x .
$$


Since $\Phi$ is area-preserving, (3.13) implies

$$
|\omega(t)|_{j} \leqslant|\omega(0)|_{j}+\int_{0}^{t}|f(s)|_{0} d s .
$$

Let $R_{j}(t)=|\omega(t)|_{j}$. If $j \leqslant 2$, we have from (3.10)

$$
R_{j}(t) \leqslant R_{j}(0)+\int_{0}^{t} C_{2} M_{3} R_{j}(s) d s .
$$

This implies $R_{j}(t) \leqslant R_{j}(0) \exp \left(C_{2} M_{3} t\right)$, which is (3.2). If $j \geqslant 3$, we know by induction that (3.3) holds with $j$ replaced by $j-1$, so that $|\omega(s)|_{j-1}$ is bounded by a constant depending only on $j, M_{3}, T_{1}$, and $|\omega(0)|_{j-1}$. Then, from (3.11),

$$
R_{j}(t) \leqslant R_{j}(0)+\int_{0}^{t} K_{j} R_{j}(s) d s,
$$

where $K_{j}$ has the same dependence. This implies (3.3).

Finally, we show that we can take $T_{2}=T_{1}$. Let $T_{*}$ be the supremum of $T_{2}<T_{1}$, so that $u$ is in the class claimed for $0 \leqslant t \leqslant T_{2}$. We show first that $u \in$ $C\left(\left[0, T_{*}\right] ; H^{m+1}\right) \cap C^{1}\left(\left[0, T_{*}\right] ; H^{m}\right)$. For $|\alpha| \leqslant m$, we now know that $f(t)$ in (3.13) is bounded in $H^{0}$ for $0 \leqslant t \leqslant T_{*}$. Thus, it follows from (3.13) that $\omega \in$ $C\left(\left[0, T_{*}\right] ; H^{m}\right)$. Then (2.13) implies that $D_{x} u$ has the same property. By writing $u$ as the integral of $u_{t}=B u$, it is clear that $u \in C\left(\left[0, T_{*}\right] ; H^{0}\right)$. Also $B u$ depends continuously in $H^{m}$ on $u \in H^{m+1}$, so $u_{t} \in C\left(\left[0, T_{*}\right] ; H^{m}\right)$.

If $T_{*}<T_{1}$, the local existence theorem of [6] would show that the solution can be continued beyond $T_{*}$ in the same class. This would contradict the definition of $T_{*}$, and therefore $T_{*}=T_{1}$. This finishes the proof of the lemma except for (3.4), which follows directly from (3.3) and (2.13).

Proof of Lemma 2.1. We use Lemmas 3.1, 3.2 and properties of $H(t)$. The operator $H(t)$ has norm $\leqslant 1$ in $L^{p}$ or $H^{s}$; moreover, for $v \in C^{\alpha}, H(t) v \in C^{\alpha}$ with the same Hölder constant. Since $H(t)$ commutes with curl, Lemma 3.1(i) implies

$$
\left|\omega_{n}\right|_{L^{\prime}}+\left|\omega_{n}\right|_{L^{\infty}} \leqslant\left|\omega_{0}\right|_{L^{\prime}}+\left|\omega_{0}\right|_{L^{\infty}}
$$

where $\omega_{n}=\nabla \times u_{n}$. Next, $\omega_{0}$ is Lipschitz continuous with constant, say, $2\left|\omega_{0}\right|_{c^{1}}$, and it follows from Lemma 3.1(ii) that $\omega_{n}$ is Hölder continuous with exponent $\beta^{n}$ and constant $2\left|\omega_{0}\right|_{C^{1}}$. Here

$$
\beta^{n}=\exp \left(-C_{0} M_{1} n \Delta t\right) \geqslant \exp \left(-C_{0} M_{1} T_{1}\right) \equiv b>0 .
$$

We may therefore apply (iv), (v) of Lemma 3.1 with $\lambda$ replaced by $b$. Using (iii)-(v), we conclude that

$$
\left|u_{n}\right|_{C^{1}}+\left|\omega_{n}\right|_{C^{1}} \leqslant M_{3}
$$

for some $M_{3}$ depending on $\left|\omega_{0}\right|_{L^{1}}+\left|\omega_{0}\right|_{C^{1}}$, which is bounded by $\left|u_{0}\right|_{X^{4}}$, and on $T$.

We can now use Lemma 3.2 to complete the proof. It follows from the above and (3.2) that $\left|\omega_{n}\right|_{2}$ is uniformly bounded. For $3 \leqslant j \leqslant s-1$, if $\left|\omega_{n}\right|_{j-1}$ is uniformly bounded, then (3.3) implies that $\left|\omega_{n}\right|_{j}$ is also. Thus, for $u_{0} \in X^{s}$, we have $\omega_{n}$ bounded in $H^{s-1}$. Since both solution operators preserve $H^{0}$-norm, $\left|u_{n}\right|_{0}=\left|u_{0}\right|_{0}$. The conclusion of the lemma now follows from (2.13).

Before proving Lemma 2.2, we derive a basic estimate. Suppose $u^{(1)}, u^{(2)}$ are divergence-free elements of $L^{\infty}\left([0, T] ; H^{s}\right)$ and $f \in L^{\infty}\left([0, T] ; H^{j}\right)$, where $3<j$ $\leqslant s-1$. Let $w$ be the solution of

$$
w_{t}+P\left(u^{(1)} \cdot \nabla w+w \nabla u^{(2)}\right)=P f, \quad \nabla \cdot w=0, \quad w(0)=w_{0} .
$$


Thus, using the $H^{j}$ inner product, we have

$$
\left|\left(w_{t}, w\right)_{j}\right| \leqslant\left|u^{(1)}\right|_{j}|w|_{j}^{2}+\left|u^{(2)}\right|_{j+1}|w|_{j}^{2}+|f|_{j}|w|_{j}
$$

The first term is estimated using (2.2) of [6]. Then for $0 \leqslant t \leqslant T$

$$
|w(t)|_{j} \leqslant C\left\{|w(0)|_{j}+\sup _{0<\tau<t}|f(\tau)|_{j}\right\},
$$

where $C$ depends only on $T$ and $\sup \left(\left|u^{(1)}(\tau)\right|_{j}+\left|u^{(2)}(\tau)\right|_{j+1}\right)$.

Proof of Lemma 2.2. It follows from Lemmas 3.1, 3.2 that $\left|F\left(t, v_{0}\right)\right|_{s}$ is bounded by a constant for $\left(t, v_{0}\right) \in K^{s}(R)$, and that $F$ is continuous in $t$ separately to $H^{s}$. Given $v_{0}, \tilde{v}_{0}$, we first estimate $F\left(t, \tilde{v}_{0}\right)-F\left(t, v_{0}\right)$. Let $v=E(t) v_{0}, \tilde{v}=E(t) \tilde{v}_{0}$, and $d=\tilde{v}-v$. Then

$$
d_{t}+P(\tilde{v} \cdot \nabla d+d \cdot \nabla v)=0
$$

and, by (3.14),

$$
|d(t)|_{s-1} \leqslant C\left|v_{0}-\tilde{v}_{0}\right|_{s-1},
$$

provided $\left(v_{0}, 0\right),\left(\tilde{v}_{0}, 0\right) \in K^{s}(R)$. It follows from this and the continuity in $t$ that $F$ is continuous from $K^{s}(R)$ to $H^{s-1}$.

We now consider the first partial derivatives with respect to $t$ and $v_{0}$. We have

$$
D_{t} F\left(t, v_{0}\right)=-P\left\{E(t) v_{0} \cdot \nabla E(t) v_{0}\right\},
$$

and according to the above remarks this is continuous from $K^{s}(R)$ to $H^{s-2}$. By a formal calculation we expect that $D_{v_{0}} F$ applied to $w_{0}$ should be the solution of

$$
w_{t}+P(v \cdot \nabla w+w \cdot \nabla v)=0, \quad \nabla \cdot w=0, \quad w(0)=w_{0} .
$$

To verify this, we set $\tilde{v}_{0}=v_{0}+w_{0}$ and define $v, \tilde{v}, d$ as before. Then $e(t)=d(t)-$ $w(t)$ satisfies

$$
e_{t}+P(v \cdot \nabla e+e \cdot \nabla v)=-P(d \cdot \nabla d), \quad \nabla \cdot e=0, \quad e(0)=0
$$

and from (3.14), (3.15)

$$
|e|_{s-2} \leqslant C|d|_{s-1}^{2} \leqslant C\left|w_{0}\right|_{s-1}^{2} .
$$

Therefore the derivative is indeed $w$ given by (3.16). We can estimate $w$ using (3.14) to show that $D_{v_{0}} F$ is bounded from $H^{s}$ to $H^{s-1}$.

We still have to show that $D_{v_{0}} F$, as an operator from $H^{s}$ to $H^{s-2}$, depends continuously on $\left(t, v_{0}\right)$. Given $v_{0}, \tilde{v}_{0}, w_{0}$, let $w$ be the solution of (3.16) with $v=E(t) v_{0}$, and let $\tilde{w}$ be the solution of the same problem with $v$ replaced by $\tilde{v}=E(t) \tilde{v}_{0}$. Let $r=\tilde{w}-w$. Then

$$
\begin{gathered}
r_{t}+P(v \cdot \nabla r+r \cdot \nabla v+d \cdot \nabla \tilde{w}+\tilde{w} \cdot \nabla d)=0, \\
\nabla \cdot r=0, \quad r(0)=0,
\end{gathered}
$$

and, by (3.14),

$$
|r|_{s-2} \leqslant C|d|_{s-1}|\tilde{w}|_{s-1} \leqslant C\left|\tilde{v}_{0}-v_{0}\right|_{s}\left|w_{0}\right|_{s-1} .
$$

Also (3.16) leads to the inequality

$$
|w(\tilde{t})-w(t)|_{s-2} \leqslant C|\tilde{t}-t|\left|w_{0}\right|_{s-1} .
$$

These last two estimates show that $w$ depends continuously on $\left(t, v_{0}\right)$, uniformly for $\left|w_{0}\right|_{s-1}$ bounded. 
It should now be clear that the statement concerning higher derivatives follows from similar arguments.

Proof of Lemma 2.3. We check by induction that

$$
\left|u_{n}\right|_{H^{\circ}} \leqslant \phi_{M}(n \Delta t) \text {. }
$$

Assuming this for given $n$, we have

$$
\left|H(\Delta t / 2) u_{n}\right|_{H^{\circ}} \leqslant \phi_{M}(n \Delta t)
$$

since $H$ has norm $\leqslant 1$. But then, from (1.5), we have

$$
\left|E(\Delta t) H(\Delta t / 2) u_{n}\right|_{H^{\circ}} \leqslant \phi_{M}((n+1) \Delta t),
$$

and therefore $u_{n+1}$ satifies the same inequality.

Lemma 2.4 follows from the proof of Lemma 2.2 and the fact that the solution is uniformly bounded in $C\left(\left[0, T_{1}\right] ; H^{\sigma}\right) \cap C^{1}\left(\left[0, T_{1}\right] ; H^{\sigma-1}\right)$, according to (1.5).

For periodic two-dimensional flows, the only difference in the argument occurs in Lemma 3.1. A construction of solutions for Euler's equations in a bounded domain, similar to that in $\mathbf{R}^{2}$ of [8], has been given by Kato [7]. It is not hard to see that this construction can also be used in the periodic case. Integrating $u_{t}$ over a fundamental domain, we find that any solution must have the property that the average value of $u(t)$ is independent of $t$. Therefore the velocity $u(t)$ is uniquely determined by the vorticity and the average value specified by $u_{0}$. The separation of the constant term causes no difficulty in the arguments. The analogue of Lemma 3.1 then follows from [7]. For three-dimensional periodic flows, we need only note that the arguments of [6] can be applied equally well to the periodic case.

4. Proof of Theorem 1.3. Our first objective is to establish the estimate in (1.8) for solutions of the Navier-Stokes equations in $\mathbf{R}^{2}$. Thus, with the notation from the previous sections, if $u^{\nu}$ satisfies

$$
u_{t}^{\nu}=B\left(u^{\nu}\right)+\nu \Delta u^{\nu}, \quad u^{\nu}(x, 0) \equiv u_{0}(x),
$$

we must prove that for any large time $T>0$, if $u_{0}$ belongs to $X^{s}$ for $s \geqslant 4$, then $u^{\nu}(t)$ belongs to $L^{\infty}\left([0, T] ; H^{s}\right)$ and there is a fixed constant $C$ so that

$$
\max _{\substack{0<t<T \\ 0<\nu<1}}\left|u^{\nu}(t)\right|_{H^{s}} \leqslant C,
$$

where $C$ depends only on $T$ and $\left|u_{0}\right|_{X^{s}}$.

Proof of the estimate (4.2). Below, $C$ always denotes a generic constant depending only on $T$ and $\left|u_{0}\right|_{X^{s}}$. When $\nu=0$, this bound has already been proved in Lemma 3.1 and Lemma 3.2. Let $u_{0}^{\varepsilon}(x)=j_{\varepsilon} * u_{0}(x)$ denote the standard mollification of the initial data in (4.1); then $u_{0}^{e}(x)$ has finite $X^{\sigma}$ norm for any $\sigma$ and

$$
\left|u_{0}^{\varepsilon}\right|_{X^{s}} \leqslant\left|u_{0}\right|_{X^{s}}, \quad u_{0}^{e} \rightarrow u_{0} \quad \text { in } X^{s} \text { as } \varepsilon>0 .
$$

Consider the solution $u^{\nu, e}$ of (4.1) with the initial data $u_{0}^{e}(x)$. From the classical existence theorem in [8] and standard Schauder estimates for the heat equation, it follows that $u^{\nu, \varepsilon}$ is a smooth function in $\mathbf{R}^{2} \times[0, T]$. Let $u_{n}^{e}$ denote the solution of the viscous splitting algorithm in (1.4) with this initial data. We define $u_{\Delta t}^{e}(t)$ as a natural interpolation of $u_{n}^{\varepsilon}$ given by

$$
u_{\Delta t}^{\varepsilon}(t)=H(\tau / 2) E(\tau) H(\tau / 2) u_{n}^{\varepsilon}
$$


for $n \Delta t \leqslant t \leqslant(n+1) \Delta t$, with $\tau=t-n \Delta t$, and any $n$ with $(n+1) \Delta t<T$. From the proof of Lemma 2.1 and (4.3), we conclude that, independent of $\Delta t$ and $\varepsilon$, $0<\varepsilon \leqslant 1$,

$$
\max _{0<t<T}\left|u_{\Delta t}^{\varepsilon}(t)\right|_{X^{s}} \leqslant C
$$

We also claim that

$$
\max _{\substack{0<t_{1}<t_{2}<T \\ 0<\varepsilon<1}}\left|u_{\Delta t}^{\varepsilon}\left(t_{1}\right)-u_{\Delta t}^{\varepsilon}\left(t_{2}\right)\right|_{L^{2}\left(\mathbf{R}^{2}\right)}<C\left|t_{1}-t_{2}\right| .
$$

For the moment, we assume (4.6) and continue the argument. Let $\rho(x)$ be a fixed smooth function with $\rho \equiv 1$ for $|x|<1$ and vanishing for $|x|>2$, and set $\rho_{R}(x)=$ $\rho(x / R)$ for $R \geqslant 1$. Below we fix $\gamma$ with $\gamma<1$ and use the well-known interpolation inequalities

$$
|u|_{s-\gamma} \leqslant C(\delta)|u|_{0}+\delta|u|_{s}, \quad \text { for any } \delta>0 \text {. }
$$

Temporarily, we fix $\varepsilon$ with $0<\varepsilon \leqslant 1$. From (4.5) and the Rellich compactness theorem, $\left\{\rho_{R} u_{\Delta t}^{\varepsilon}(t): \Delta t=T / N, N \geqslant 1\right\}$ is a precompact subset of $L^{2}\left(\mathbf{R}^{2}\right)$ for any fixed $t$. Therefore the estimates (4.5), (4.6) allow us to apply the proof of the Arzela-Ascoli theorem and conclude that $\left\{\rho_{R} u_{\Delta t}^{e}(t)\right\}$ is precompact in $C\left([0, T] ; L^{2}\right)$. As in the proof of the Lions-Aubin Lemma (e.g., see [12, Theorem III.2.1, pp. 271-272]), we use (4.7) to strengthen this conclusion to

$$
\left\{\rho_{R} u_{\Delta t}^{e}(t)\right\} \text { is precompact in } C\left([0, T] ; H^{s-\gamma}\right) .
$$

However, for fixed $\varepsilon, u_{0}^{e}$ also belongs to $X^{\sigma}$; thus it follows from Theorem 1.1, (4.8), and the uniqueness of limits in $C\left([0, T] ; L^{2}\right)$ that

$$
\rho_{R} u_{\Delta t}^{\varepsilon}(t) \rightarrow \rho_{R} u^{\nu, \varepsilon}(t) \quad \text { in } C\left([0, T] ; H^{s-\gamma}\right) \text { as } \Delta t \rightarrow 0 .
$$

By passing to the limit, we see that (4.9) implies that $\rho_{R} u^{\nu, e}$ inherits the bounds in (4.5) and (4.6) with slightly larger constants $C$ independent of $R>1$ and $\varepsilon<1$. By letting $R$ approach infinity, we conclude that the estimate

$$
\begin{gathered}
\max _{0<t<T}\left|u^{\nu, e}(t)\right|_{X^{s}} \leqslant C, \\
\max _{0<t_{1}<t_{2}<T}\left|u^{\nu, \varepsilon}\left(t_{1}\right)-u^{\nu, e}\left(t_{2}\right)\right|_{L^{2}}<C\left|t_{1}-t_{2}\right|
\end{gathered}
$$

are valid independent of $\nu$ and $\varepsilon$ for $0<\nu<1$ and $0<\varepsilon<1$.

Recall that $u^{\nu, \varepsilon}$ satisfies (4.1) so that

$$
u_{t}^{\nu, \varepsilon}=B\left(u^{\nu, \varepsilon}\right)+\nu \Delta u^{\nu, \varepsilon} \text {. }
$$

Integration of this identity from $t_{1}$ to $t_{2}$, the use of the first inequality in (4.10a), and the fact that $s \geqslant 4$ (see [6]) implies the improved Lipschitz estimate,

$$
\max _{\substack{0<t_{1}<t_{2}<T \\ 0<\nu<1 \\ 0<\varepsilon<1}}\left|u^{\nu, \varepsilon}\left(t_{1}\right)-u^{\nu, \varepsilon}\left(t_{2}\right)\right|_{H^{2}} \leqslant C\left|t_{1}-t_{2}\right| .
$$

Next, we let $\varepsilon \rightarrow 0$; using (4.10a,b), we apply the argument preceding (4.8) to $\rho_{R} u^{\nu, e}$. By choosing a diagonal subsequence, we find that there exists $\varepsilon_{j} \rightarrow 0$ and a function $\tilde{u}(t)$ so that

$$
u^{\nu, \xi_{j}} \rightarrow \tilde{u} \quad \text { in } C\left([0, T] ; H_{\text {loc }}^{s-\gamma}\right) \text { as } \varepsilon_{j} \rightarrow 0
$$


and

$$
\left|\tilde{u}\left(t_{1}\right)-\tilde{u}\left(t_{2}\right)\right|_{H^{2}}<C\left|t_{1}-t_{2}\right| .
$$

Moreover, from the weak compactness of bounded sets in $H^{s}$, we have

$$
\max _{0<t<T}|\tilde{u}(t)|_{s}<C,
$$

and it follows from (4.11a) and (4.7) that $\tilde{u} \in C\left([0, T] ; H^{s-\gamma}\right)$. In (4.11a,b) $C$ is the constant from (4.10). Since $u^{\nu, \xi}$ is a solution of (4.1) for each $\varepsilon_{j}$, and since $s-\gamma \geqslant 3$, we see from (4.3), (4.11) that $\tilde{u}$ is a generalized solution of (4.1) in the sense defined in [6] so that $\tilde{u} \in C\left([0, T] ; H^{3}\right) \cap A C\left([0, T] ; H^{2}\right) \cap L^{1}\left([0, T] ; H^{4}\right)$. By the uniqueness theorem of [6], $\tilde{u} \equiv u^{\nu}$ where $u^{\nu}$ is the solution of (4.1) with initial data $u_{0}(x)$ (actually, we have used the above argument to construct this solution), and the estimate (4.11b) implies the required estimate in (4.2).

It remains to prove (4.6). It is obviously sufficient to check this estimate for $t_{1}, t_{2}$ satisfying $n \Delta t \leqslant t_{1}<t_{2} \leqslant(n+1) \Delta t$. With $\tau_{j}=t_{j}-n \Delta t$, we have by the triangle inequality,

$$
\begin{aligned}
\left|H\left(\frac{\tau_{1}}{2}\right) E\left(\tau_{1}\right) H\left(\frac{\tau_{1}}{2}\right) u_{n}^{e}-H\left(\frac{\tau_{2}}{2}\right) E\left(\tau_{2}\right) H\left(\frac{\tau_{2}}{2}\right) u_{n}^{e}\right|_{L^{2}} \\
\leq\left|H\left(\frac{\tau_{1}}{2}\right) E\left(\tau_{1}\right) H\left(\frac{\tau_{1}}{2}\right) u_{n}^{e}-H\left(\frac{\tau_{2}}{2}\right) E\left(\tau_{1}\right) H\left(\frac{\tau_{1}}{2}\right) u_{n}^{e}\right|_{L^{2}} \\
+\left|E\left(\tau_{1}\right) H\left(\frac{\tau_{1}}{2}\right) u_{n}^{e}-E\left(\tau_{2}\right) H\left(\frac{\tau_{1}}{2}\right) u_{n}^{e}\right|_{L^{2}} \\
+\left|E\left(\tau_{2}\right) H\left(\frac{\tau_{1}}{2}\right) u_{n}^{e}-E\left(\tau_{2}\right) H\left(\frac{\tau_{2}}{2}\right) u_{n}^{e}\right|_{L^{2}}
\end{aligned}
$$

From Lemma 2.1, we have $\left|u_{n}^{e}\right|_{X^{s}}<C$, so that, by Lemma 3.2,

$$
\max _{\substack{0<s_{1}, s_{2}<\Delta t / 2 \\ 0<t<\Delta t}}\left|H\left(s_{1}\right) E(t) H\left(s_{2}\right) u_{n}^{e}\right|_{X^{s}}<C .
$$

This fact implies the bound

$$
\left|\nu \Delta H(s) E\left(\tau_{1}\right) H\left(\tau_{1} / 2\right) u_{n}^{\varepsilon}\right|_{L^{2}}<C \nu
$$

applying this estimate and the formula

$$
H\left(\tau_{2} / 2\right)-H\left(\tau_{1} / 2\right)=\nu \int_{\tau_{1} / 2}^{\tau_{2} / 2} \Delta H(s) d s,
$$

we see that the first term on the right-hand side of (4.12) is bounded by $\nu C\left|t_{1}-t_{2}\right|$. The second term on the right in (4.12) is similarly estimated by using (4.13), the formula

$$
E\left(\tau_{1}\right) v-E\left(\tau_{2}\right) v=\int_{\tau_{2}}^{\tau_{1}} B(E(s) v) d s,
$$

and the fact that $H^{s}$ is a Banach algebra under multiplication for $s \geqslant 2$; thus, the second term on the right in (4.12) is bounded by $C\left|t_{1}-t_{2}\right|$. Finally, for the third term above, we use the simplest $L^{2}$-energy estimate mentioned in the discussion above (3.14) to bound this term by

$$
C\left|H\left(\tau_{1} / 2\right) u_{n}^{e}-H\left(\tau_{2} / 2\right) u_{n}^{e}\right|_{L^{2}}
$$

By using (4.13) and (4.14) once again, we bound this by $C \nu\left|t_{1}-t_{2}\right|$. This establishes the fact in (4.6). 
It should already be evident to the reader that, once the bound in (4.2) is established, the argument below Lemma 2.3 and Lemma 2.4 can be repeated with no essential changes to yield the second statement in Theorem 1.3. To prove the first statement in the theorem, we set $r=u^{\nu}-u^{0}$ and observe that in the argument below Lemma 2.3 and Lemma 2.4, we can set $f=\nu \Delta u^{0}$. Thus, if $u_{0}(x)$ belongs to $X^{s}$, from Lemma 3.1,

$$
|f|_{s-2}=\left|\nu \Delta u^{0}\right|_{s-2} \leqslant \nu C .
$$

With this remark, the same argument can be repeated with only minor changes to prove the first statement of Theorem 1.3. Of course, statement (3) for part (B) of the main theorem follows in the same fashion for short times when $N=3$.

Department of Mathematics

Tulane University

New Orleans, Louisiana 70118

Department of Mathematics

University of California

Berkeley, California 94720

1. G. K. BAtchelor, An Introduction to Fluid Mechanics, Cambridge Univ. Press, New York, 1970.

2. A. J. Chorin, "Numerical study of slightly viscous flow," J. Fluid Mech., v. 57, 1973, pp. $785-796$.

3. A. J. Chorin, T. Hughes, M. McCracken \& J. Marsden, "Product formulas and numerical algorithms," Comm. Pure Appl. Math., v. 31, 1978, pp. $205-256$.

4. D. EBIN \& J. MARSDEN, "Groups of diffeomorphisms and the motion of an incompressible fluid," Ann. of Math., v. 92, 1970, pp. 102-163.

5. O. HALD, "Convergence of vortex methods for Euler's equations. II," SIAM J. Numer. Anal., v. 16, 1979, pp. 726-755.

6. T. KAto, "Nonstationary flows of viscous and ideal fluids in $\mathbf{R}^{3}$," J. Funct. Anal., v. 9, 1972, pp. 296-305.

7. T. KAto, "On classical solutions of the two-dimensional Euler equation," Arch. Rational Mech. Anal., v. 25, 1967, pp. 188-200.

8. F. J. McGrath, "Nonstationary plane flow of viscous and ideal fluids," Arch. Rational Mech. Anal., v. 27, 1968, pp. 329-348.

9. F. Milinazzo \& P. G. Saffman, "The calculation of large Reynolds number two-dimensional flow using discrete vortices with random walk," J. Comput. Phys., v. 23, 1977, pp. 380-392.

10. G. Strang, "On the construction and comparison of difference schemes," SIAM J. Numer. Anal., v. 5, 1968, pp. 506-517.

11. G. Strang, "Accurate partial difference methods. II. Nonlinear problems," Numer. Math., v. 6, 1964, pp. 37-49.

12. R. Temaм, The Navier-Stokes Equations, North-Holland, Amsterdam, 1977. 